\title{
MANIFOLDS ON WHICH ONLY TORI CAN ACT
}

\author{
KYUNG BAI LEE AND FRANK RAYMOND
}

Dedicated to Professor E. E. Floyd

\begin{abstract}
A list of various types of connected, closed oriented manifolds are given. Each of the manifolds support some of the well-known compact transformation group properties enjoyed by aspherical manifolds. We list and describe these classes and their transformation group properties in increasing generality. We show by various examples that these implications can never be reversed. This establishes a hierarchy in terms of spaces in one direction and the properties they enjoy in the opposite direction.
\end{abstract}

1. Introduction. The following theorem was proved by Conner and Raymond in 1967 [CR1].

THEOREM. The only connected, compact Lie groups that act effectively on a closed aspherical manifold $M$ are tori. Moreover, the dimension of $G$ is not greater than the rank of the center of $\pi_{1}(M)$.

Subsequently, there were various generalizations obtained by similar methods and different methods. For example, Conner-Raymond [CR3, CR5], Schoen-Yau [SY], Donnelly-Schultz [DS], Ku-Ku [KK], Browder-Hsiang [BH], Washiyama-Watabe [WW], Gottlieb-Lee-Ozaydin [GLO] and others. Theorems for finite groups acting on aspherical manifolds obtained in [CR1] were also generalized in many articles; e.g., [CR2, LY, Sch1, 2, DS, B1, AB, GLO, SY and LR2]. In particular, the technique to extend transformation group results to certain larger classes by mapping them into $K(\pi, 1)$ 's was introduced by Schoen and Yau.

As the various authors enlarged the class of spaces which enjoyed some of the features of compact groups acting on aspherical manifolds, there arose the question as to what were the actual interrelationships among these new classes of spaces and manifolds and the different results proved about them. In this paper we list most of the new classes and the transformation group properties possessed by them in increasing generality. We show that the implications can never be reversed. This

Received by the editors May 1, 1986.

1980 Mathematics Subject Classification (1985 Revision). Primary 57S10; Secondary 57N99.

Key words and phrases. Compact transformation group, aspherical manifold, covering space, ends, $K$-manifold, $K(\pi, 1)$, essential manifold, admissible, injective action, inner action, compact Lie group, hyperaspherical manifold, lens space, spherical space form, toral action.

Research of the first author supported in part by NSF Grant MCS 8201033.

Research of the second author supported in part by NSF Grant 8120790 . 
establishes a sort of hierarchy in terms of the spaces involved in one direction and the properties they enjoy in the opposite direction. Here are the relevant definitions.

A connected, closed, oriented m-manifold $M$ is called:

1. Aspherical if $\pi_{i}(M)=0$, for all $i>1$. $M$ is therefore a $K(\pi, 1)$, where $\pi=\pi_{1}(M)$.

2. Hyperaspherical [DS] if there exists a closed aspherical $m$-manifold $N$ and a map $f: M \rightarrow N$ of degree 1 . That is, $f^{*}: H^{m}(N ; \mathbf{Z}) \rightarrow H^{m}(M ; \mathbf{Z})$ is onto.

3. K-manifold [GLO] if there exists a torsion-free group $\Gamma$ and a map $f$ : $M \rightarrow K(\Gamma, 1)$ so that $f^{*}: H^{m}(K(\Gamma, 1), \mathbf{Z}) \rightarrow H^{m}(M, \mathbf{Z})$ is onto.

4. We may also add that $M$ is a rational $K$-manifold if we replace the $f^{*}$ above by $f^{*}: H^{m}(K(\Gamma, 1) ; \mathbf{Q}) \rightarrow H^{m}(M ; \mathbf{Q})$. Obviously, a $K$-manifold is a rational $K$-manifold.

5. Essential [G] if $c^{*}: H^{m}(K(\pi, 1) ; \mathbf{Q}) \rightarrow H^{m}(M ; \mathbf{Q})$ is nontrivial, where $c$ : $M \rightarrow K(\pi, 1)$ is a classifying map $\left(\pi=\pi_{1}(M)\right)$.

6. Admissible [LR1] if the only periodic self-homeomorphisms of $\tilde{M}$ (the universal covering of $M)$ commuting with $\pi=\pi_{1}(M)$ are elements of the center of $\pi, Z(\pi)$.

An action of a compact Lie group $G$ on $M$ is called:

(i) Injective [CR3] if $G$ is connected and $\mathrm{ev}_{*}^{x}: \pi_{1}(G, e) \rightarrow \pi_{1}(M, x)$ is an injective homomorphism, where the evaluation map is defined by $\operatorname{ev}^{x}(g)=g x$.

(ii) Inner [GLO] if $G$ induces the trivial homomorphism of $G$ into Out $\pi$. (Out $\pi=$ Automorphism of $\pi_{1}(M)$ modulo the inner automorphism of $\pi_{1}(M)$ ).) For example, if $G$ is connected, then $g: M \rightarrow M$ is isotopic to the identity for any $g \in G$.

\section{Statement of results.}

THEOREM 1. For $M$ a connected, oriented, closed m-manifold the following implications hold:

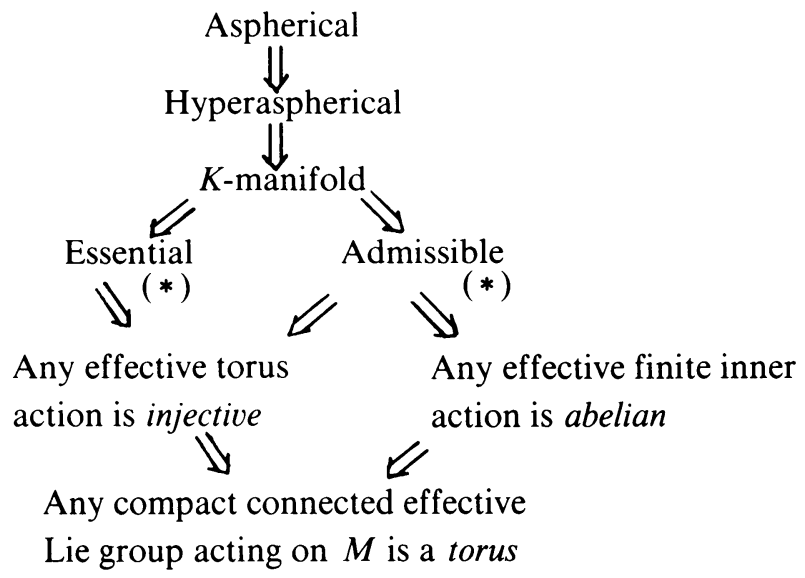

THEOREM 2. None of the implications in Theorem 1 can be reversed.

(*) The theorems as given are topological statements but the arguments need smoothness in two places: Essential $\Rightarrow$ Injective and Inner is abelian $\Rightarrow$ Admissible. We do not know if the purely topological statements are valid in both of these instances. 
In the proofs of the theorems we shall interpolate even more classes in order to exhibit as fine a tuning as we presently understand. We have not included them in the statements of the theorems as we wanted to keep these statements as palatable as possible. In $\S 5$ we shall give a new diagram which summarizes what we actually do prove. We wish to thank F. T. Farrell for his help with (4.3).

The second-named author presented some of the contents of this paper at a Symposium at the University of Virginia, April 1984, which honored the contributions of Professor E. E. Floyd to Topology.

\section{Proof of Theorem 1.}

3.1. We note first the following:

$$
\begin{aligned}
& \text { Aspherical } \Rightarrow \text { Injective } \Rightarrow \text { Torus } \quad[\text { CR1], } \\
& \text { Essential } \Rightarrow \text { Smoothly injective (see 3.4) } \quad[\text { BH], } \\
& \text { (Rationally) Hyperaspherical } \Rightarrow \text { Injective [DS], } \\
& \text { (Rational) } K \text {-manifold } \Rightarrow \text { Injective } \quad[W W], \\
& K \text {-manifold } \Rightarrow \text { Abelian } \Rightarrow \text { Torus } \quad \text { [GLO]. }
\end{aligned}
$$

In [WW] the argument is actually stated for Hyperaspherical $\Rightarrow$ Injective, but the argument given is valid as stated above. The reader should observe, however, that the proof for Lemma 1 given there is not quite correct but their claim is still correct.

Notation. Let $A$ be a subgroup of $\pi$. Then $C_{\pi}(A), Z(\pi), t(\pi)$ denote the centralizer of $A$ in $\pi$, the center of $\pi$, the normal subgroup generated by the set of all torsion elements of $\pi$, respectively. For a space $M$, the universal covering is denoted by $\widetilde{M}, \mathscr{H}(M)$ denotes the group of self-homeomorphisms of $M$.

3.2. $K$-manifold $\Rightarrow$ Admissible. Suppose $M$ is a $K$-manifold which is not admissible. Then there exists a homeomorphism $h$ of $\tilde{M}$ so that

(i) $h$ commutes with $\pi$,

(ii) $h^{k}=$ id, for some $k>1$,

(iii) $h \notin Z(\pi)$, the center of $\pi$.

Let $p$ the be smallest integer so that $h^{p} \in Z(\pi), 1<p \leqslant k$. Let $k=d \cdot p$. We may assume $p$ is a prime by choosing a power of $h$ if necessary. Then

$$
\mathbf{Z}_{k}=\left\{h, h^{2}, \ldots, h^{k}\right\} \subset C_{\mathscr{H}(\tilde{M})}(\pi),
$$

the centralizer of $\pi$ in $\mathscr{H}(\tilde{M})$,

$$
\mathbf{Z}_{d}=\left\{h^{p}, h^{2 p}, \ldots, h^{d p}\right\}=\mathbf{Z}_{k} \cap \pi=\mathbf{Z}_{k} \cap Z(\pi) .
$$

Then such an $h$ defines an action of $\mathbf{Z}_{p} \cong \mathbf{Z}_{k} / \mathbf{Z}_{d}$ on $M$. The lifting sequence of $\left(\mathbf{Z}_{p}, M\right)$ is $1 \rightarrow \pi \stackrel{i}{\rightarrow} E \rightarrow \mathbf{Z}_{p} \rightarrow 1$ and $\pi \supset Z(\pi) \supset \mathbf{Z}_{d}, E \supset C_{E}(\pi) \supset \mathbf{Z}_{k}$ so that $1 \rightarrow \mathbf{Z}_{d} \rightarrow \mathbf{Z}_{k} \rightarrow \mathbf{Z}_{p} \rightarrow 1$ is exact.

Assume $\operatorname{Fix}\left(\mathbf{Z}_{p}, M\right)=\varnothing$. Then $\pi_{1}\left(M / \mathbf{Z}_{p}\right)=E$. The set of torsion elements of $C_{E}(\pi)$ forms a fully invariant subgroup of $C_{E}(\pi)$ coinciding with $t C_{E}(\pi)$ and

$$
1 \rightarrow t(Z(\pi)) \rightarrow t\left(C_{E}(\pi)\right) \rightarrow \mathbf{Z}_{p} \rightarrow 1
$$


is exact [GLO, 1.2]. Then $\pi / t(Z(\pi)) \cong E / t\left(C_{E}(\pi)\right)$. The kernel of the homomorphism $\pi \stackrel{f_{\sharp}}{\rightarrow} \Gamma$, induced from $f: M \rightarrow K(\Gamma, 1)$ contains the smallest normal subgroup containing all the torsion of $\pi$. Therefore $\pi \rightarrow \Gamma$ factors through $\pi / t(Z(\pi))$. Consequently, we may extend the homomorphism $\pi \rightarrow \Gamma$ to $E \rightarrow \Gamma$ via

$$
\begin{gathered}
\pi \rightarrow \pi / t(Z(\pi))=E / t\left(C_{E}(\pi)\right) \rightarrow \Gamma \\
\qquad \cong \pi_{1}\left(M / \mathbf{Z}_{p}\right) .
\end{gathered}
$$

If $\operatorname{Fix}\left(\mathbf{Z}_{p}, M\right) \neq \varnothing$, then $E \cong \pi \times \mathbf{Z}_{p}$, where $\mathbf{Z}_{p}$ is the stabilizer of $E$ at a preimage of a fixed point. Now $\pi_{1}\left(M / \mathbf{Z}_{p}\right) \cong E / N$, where $N$ is the smallest normal subgroup containing all the stabilizers $[\mathbf{A}]$. Since $\mathbf{Z}_{p} \subset N$ already, $\pi_{1}\left(M / \mathbf{Z}_{p}\right)$ is a quotient of $\pi$ by a normal subgroup of $\pi$ generated by torsion elements. Thus the homomorphism $\pi \rightarrow \Gamma$ again factors through $\pi_{1}\left(M / \mathbf{Z}_{p}\right) \rightarrow \Gamma$.

In either case, we have

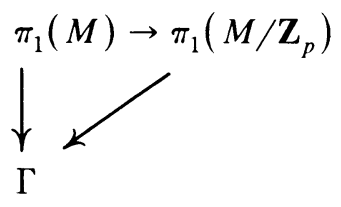

This induces a homotopy commutative diagram

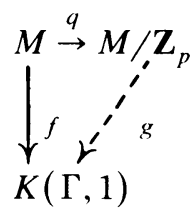

where $q$ is the orbit mapping. The map $g$ can be constructed [Sp, p. 428] because $M / \mathbf{Z}_{p}$ has the homotopy type of a CW-complex since Floyd has shown that $M / \mathbf{Z}_{p}$ is an ANR [F]. The induced diagram on cohomology $f^{*}=q^{*} \circ g^{*}$ in dimension $m_{q^{*}}$ leads to a contradiction, for it was assumed that $f^{*}$ was onto, but $H^{m}\left(M / \mathbf{Z}_{p} ; \mathbf{Z}\right) \stackrel{q^{*}}{\rightarrow}$ $H^{m}(M ; \mathbf{Z})$ is never onto [DS, Lemma 2.5].

3.3. Admissible $\Rightarrow$ Any finite inner action is abelian. Let $G$ be a finite inner action. Then

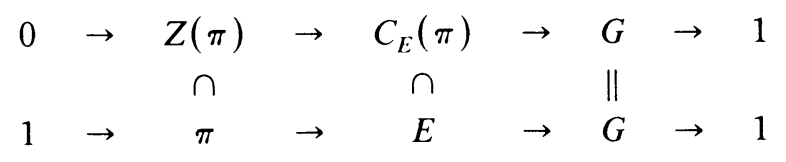

are exact. Admissibility implies that $t(Z(\pi))=t\left(C_{E}(\pi)\right)$ since admissiblity is equivalent to $t(Z(\pi))=t\left(C_{\mathscr{H}(\tilde{M})}(\pi)\right)$. Clearly $C_{E}(\pi) / t(Z(\pi))$ is torsion free, so we 
obtain

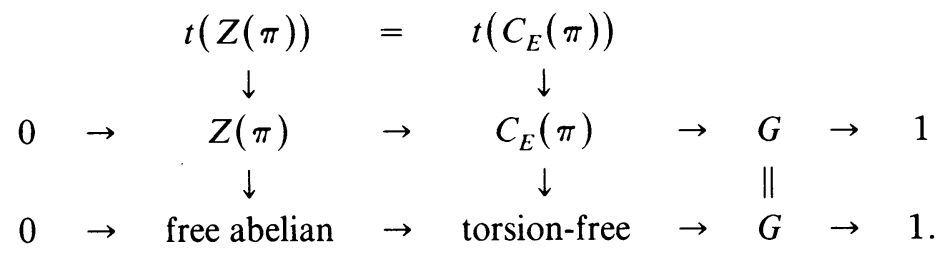

By a stronger version of [LR1, Fact 2] in [GLO] (without finite generation of the free abelian group), the middle group of the bottom sequence is abelian. Therefore, $G$ i s abelian. This proof is essentially in [GLO].

3.4. Essential or Admissible $\Rightarrow$ Injective. The argument that Essential implies Injective as given by Browder and Hsiang [BH] assumes that the action is smooth. They state that their constructions and theorems work for topological actions which can be equivariantly embedded in a smooth manifold such that the embedding admits a smooth regular neighborhood. Unfortunately, we do not know a proof for the purely topological implication without making some additional assumptions. The following will suffice as we shall see:

A connected manifold $M$ is called Z-essential if there exists a classifying map $f$ : $M \rightarrow K(\Gamma, 1)$ (with $\Gamma \cong \pi_{1}(M)$ not necessarily torsion free), so that $f^{*}$ : $H^{m}(K(\Gamma, 1) ; \mathbf{Z}) \rightarrow H^{m}(M ; \mathbf{Z})$ is nontrivial. If $H_{m}\left(K\left(\pi_{1}(M) ; \mathbf{Z}\right)\right)$ is finitely generated, then $M$ is $\mathbf{Z}$-essential if and only if $M$ is essential.

To derive the topological conclusion we postulate that $M$ is $\mathbf{Z}$-essential and a mild additional condition on $\pi_{1}(M)$ :

We say that $M$ is strongly $\mathbf{Z}$-essential if $M$ is $\mathbf{Z}$-essential and there exists infinitely many primes $p$ so that $\pi_{1}(M)$ has no elements of order $p$.

We shall show

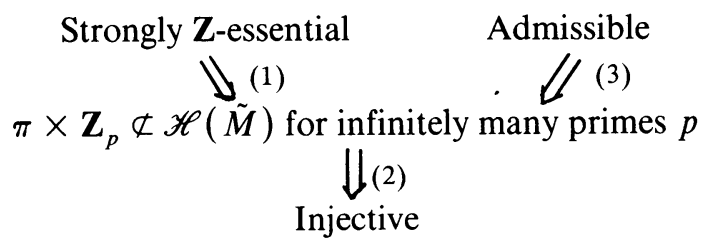

(1) Assume $M$ is strongly $\mathbf{Z}$-essential. Suppose $\pi \times \mathbf{Z}_{p} \subset \mathscr{H}(\tilde{M})$ for almost all primes $p$. Choose such a prime $p$ bigger than $d$, where $d \mathbf{Z}=$ image of $f^{*}$ : $H^{m}(K(\Gamma, 1) ; \mathbf{Z}) \rightarrow H^{m}(M ; \mathbf{Z})$. By our hypothesis, one may assume that $\pi$ contains no element of order $p$. Then such a $\mathbf{Z}_{p} \subset \mathscr{H}(\tilde{M})$ induces an action of $\mathbf{Z}_{p}$ on $M$, and $\pi_{1}\left(M / \mathbf{Z}_{p}\right)$ is either $\pi \times \mathbf{Z}_{p}$ or $\pi$, depending on whether the action is free or not. Then one can factor $f: M \rightarrow K(\Gamma, 1)$, up to homotopy, through $M / \mathbf{Z}_{p}$ as before. But $q^{*}: H^{m}\left(M / \mathbf{Z}_{p} ; \mathbf{Z}\right) \rightarrow H^{m}(M ; \mathbf{Z})$ is multiplication by $p$ if $\mathbf{Z}_{p}$ preserves orientation and if $p=2$ and reverses orientation, $q^{*}$ is trivial. But, $p>d$ and we get a contradiction. Q.E.D.

(2) Suppose there is an effective action of $S^{1}$ which is not acting injectively. Lift $S^{1}$, or a finite cover ' $S^{1}$ of $S^{1}$, to the universal covering $\tilde{M}$ of $M$. Then ' $S^{1}$ commutes with the covering transformations. $\pi \times{ }^{\prime} S^{1}$ may not be effective, but all but a finite 
number of primes $p$ satisfy $\mathbf{Z}_{p} \subset^{\prime} S^{1}$ and $\mathbf{Z}_{p} \times \pi$ acts effectively on $\tilde{M}$. (We just need to avoid the primes that divide the order of the stability groups of $S^{1}$ on $M$ and the primes that divide the order of the image of $\mathrm{ev}_{*}^{x}: \pi_{1}\left(S^{1}, 1\right) \rightarrow \pi_{1}(M, x)$.)

(3) Obviously, Admissible implies that $\pi \times \mathbf{Z}_{p} \not \subset \mathscr{H}(\tilde{M})$, for all primes $p$, and so (3) is clear.

3.5. Let us call a connected closed oriented manifold $M$ weakly admissible if $\pi \times \mathbf{Z}_{p} \not \subset \mathscr{H}(\hat{M})$, for all primes $p$. Also, $M$ is called almost weakly admissible if $\pi \times \mathbf{Z}_{p} \not \subset \mathscr{H}(\hat{M})$ for an infinite number of primes $p$. Therefore,

$M$ is weakly admissible iff the lifts of any inner compact action of $G$ to $\tilde{M}$ never contain $Z(\pi) \times G$.

COROllary. If $M$ is weakly admissible and $G$ is a compact Lie group which acts effectively, then

(i) $Z\left(\pi_{1}(M)\right)=1$ implies $G$ is finite and $\Psi: G \rightarrow$ Out $\pi_{1}(M)$ is a monomorphism.

(ii) $\operatorname{Fix}(G, M) \neq \varnothing$ implies $G$ is finite and $\theta: G \rightarrow \operatorname{Aut}\left(\pi_{1}(M)\right)$ is a monomorphism.

Here $\Psi$ is the abstract kernel induced from the lifting sequence $1 \rightarrow \pi \rightarrow E \rightarrow G$ $\rightarrow 1$ and $\theta$ is the representation into $\operatorname{Aut}\left(\pi_{1}(M)\right)$ when a base point fixed by $G$ is chosen. The corollary extends well-known results of [CR1, 2, SY, DS and GLO].

\section{Proof of Theorem 2.}

4.1. Hyperaspherical $\nRightarrow$ aspherical. Let $M=N_{1} \# N_{2}$ where $N_{1}$ is a closed oriented aspherical manifold and $N_{2}$ is any manifold other than a homotopy sphere. Then $M$ is not aspherical but it is hyperaspherical, if the dimension is bigger than 2 . (Just map $M$ to $N_{1}$ by "collapsing $N_{2}$ to a point".) Also products of hyperaspherical manifolds are hyperaspherical again.

If $M \stackrel{f}{\rightarrow} K(\Gamma, 1)$ is such that $H^{m}(K(\Gamma, 1)) \rightarrow H^{m}(M)$ is nontrivial $(\mathbf{Z}$ or $\mathbf{Q}$ coefficients), then we can assume, without any loss of generality, $f_{\#}: \pi_{1}(M) \rightarrow \Gamma$ is onto. For, if not one just passes to the covering $K_{H}$ of $K(\Gamma, 1)$ associated to the image $\left(f_{\#}\right)=H \subset \Gamma$. Then there exists a lift $\tilde{f}: M \rightarrow K_{H}$ so that $p \circ \tilde{f}=f$, where $p: K_{H} \rightarrow K$ is the covering projection. Then $f^{*}=\tilde{f}^{*} \circ p^{*}$ on cohomology which implies $\tilde{f}^{*}$ is nontrivial (respectively, onto) if $f^{*}$ is nontrivial (respectively, onto). Moreover, we may factor $f: M \rightarrow K(\Gamma, 1)$ through $K\left(\pi_{1}(M), 1\right)$. For, we take any characteristic map $c: M \rightarrow K\left(\pi_{1}(M), 1\right)$ induced by an isomorphism and we may find $g: K\left(\pi_{1}(M), 1\right) \rightarrow K(\Gamma, 1)$ so that $g \circ c \sim f$. Therefore, $c^{*}$ must be nontrivial (respectively, onto) if $f^{*}$ is.

\section{3. $K$-manifold $\nRightarrow$ rationally hyperaspherical.}

EXAMPLE. There exist $K$-manifolds $M^{m}$ which are not rationally hyperaspherical.

The examples are a modification of an example generously suggested to us by F. T. Farrell. By the Baumslag-Dyer-Heller [BDH] refinement of the Kan-Thurston construction, there is a finitely presented group $\pi$ and a continuous map $\varphi$ : $K(\pi, 1) \rightarrow \mathbf{C} P_{n}$ inducing an isomorphism on $\mathbf{Z}$-homology. In fact, as $\mathbf{C} P_{n}$ is a finite 
complex, the complex $K=K(\pi, 1)$ can be chosen to be a finite complex and hence $\pi$ must be torsion-free. Let $\alpha \in H^{2}(\pi ; \mathbf{Z})$ generate the $\mathbf{Z}$-cohomology ring. Choose $m=2 s$ even so that $6 \leqslant m \leqslant n$. By Steenrod representability (see e.g. [C, §15.2]), there exists a map from some closed oriented smooth manifold $M^{m} \stackrel{f}{\rightarrow} K$ so that $f^{*}\left[\alpha^{s}\right]=[M]$. By elementary surgery, we may assume, without any loss of generality, that $f_{\#}: \pi_{1}\left(M^{m}\right) \rightarrow \pi=\pi_{1}(K)$ is an isomorphism. (For, if it fails to be onto, we may add connected sums of $S^{1} \times S^{m-1}$ to $M^{m}$ and "extend" the mapping $f$ to $F$ with $F^{*}\left[\alpha^{s}\right]=\left[M^{\prime}\right]=\left[M \# S^{1} \times S^{m-1} \# \cdots \# S^{1} \times S^{m-1}\right]$. We then may proceed to kill the kernel of $F_{\#}$ and modify the map $F$ to $G$ from the surgered $M^{\prime}$ to $K$ with $G^{*}\left[\alpha^{s}\right]=$ [surgered $\left.M^{\prime}\right]$.)

We claim that $M$ is not rationally hyperaspherical. For suppose there exists $g$ : $M \rightarrow N^{m}$ so that $N$ is a closed aspherical manifold with $g^{*}: H^{m}(N ; \mathbf{Q}) \rightarrow$ $H^{m}\left(M\right.$; Q) nontrivial. We can assume that $g_{\#}: \pi_{1}(M) \rightarrow \pi_{1}(N)$ is surjective for, if not, the mapping $g: M \rightarrow N$ may be factored through a covering $N^{\prime}$ where $\pi_{1}\left(N^{\prime}\right)=$ image $g_{*}\left(\pi_{1}(M)\right)$. Then, there exists a map $c: K \rightarrow N$ so that $c \circ f \sim g$. Consequently, $g *(H(N ; \mathbf{Q}))=f^{*}\left(c^{*} H^{m}(N ; \mathbf{Q})\right) \cong \mathbf{Q}$. So choose a generator $\gamma \in$ $H^{m}(N ; \mathbf{Q})$ then $c^{*}(\gamma)=a \alpha^{s}$ for some rational $a$. Then $c^{*}(\gamma \cup \gamma)=c^{*}(\gamma) \cup c^{*}(\gamma)$ $=a^{2} \alpha^{2 s}$, which is a nonzero element of $H^{2 m}(\pi ; \mathbf{Q})$. But, $\gamma \cup \gamma=0$, a contradiction.

\subsection{Z-Essential $\nRightarrow$ Rational $K$-manifold.}

EXAMPLE. There exist closed oriented manifolds $M^{m}$ such that the classifying maps $c$ : $M \rightarrow K(\pi, 1)$ induce nontrivial homomorphisms $c^{*}: H^{m}(K(\pi, 1), \mathbf{Z}) \rightarrow H^{m}(M ; \mathbf{Z})$ but are not rational $K$-manifolds.

Let $\pi$ be a finitely presented group so that $\pi$ is normally generated by finite subgroups and $H_{s}(\pi ; \mathbf{Z})$ has no odd torsion, and is finitely generated for each $s$, then each element of $H_{s}(\pi ; \mathbf{Z})$ is Steenrod representable. See P. Conner [C, 15.2].

Let $M^{m} \stackrel{\jmath}{\rightarrow} K(\pi, 1)=K$ be a Steenrod representable map for some class in $H_{m}(K ; \mathbf{Z})$ of infinite order. We assume $m \geqslant 6$. We then do surgery to make $f_{\#}$ : $\pi_{1}\left(M^{m}\right) \rightarrow \pi$ an isomorphism and while modifying the map $f$ we still keep the new $M^{m}$ Steenrod representable. For example, choose $m=6$ and for $\pi$ we can choose $Q \times Q \times Q$ where $Q$ is a cocompact Fuchsian group for which $\mathbf{H} / Q$ is the 2-sphere and such that the orders of the finite subgroups of $Q$ are all powers of 2 . Then $H_{2}(Q ; \mathbf{Z}) \cong \mathbf{Z}, H_{6}(\pi ; \mathbf{Z}) \cong \mathbf{Z} \oplus 2$-torsion (the torsion of $H_{*}(\pi ; \mathbf{Z})$ is just 2-torsion). Now for $\Gamma$ torsion-free, $\pi \rightarrow \Gamma$ is always trivial since the kernel $\pi$ must contain all the torsion subgroups of $\pi$. But the smallest normal subgroup of $\pi$ that contains all the torsion of $\pi$ is $\pi$ itself because this is also true for $Q$. So, for any $g$ : $M \rightarrow K(\Gamma, 1), g$ factors through $\pi_{1}(M) / t(\pi)=1$. Hence $g$ is homotopic to a constant map. In particular, this means that there exists no $f: M \rightarrow K(\Gamma, 1)$ where $\Gamma$ is torsion-free and $H^{m}(K(\Gamma, 1) ; \mathbf{Q}) \rightarrow H^{m}(M ; \mathbf{Q})$ is nontrivial. Q.E.D.

4.5. RemarKs. (i) Obviously, this construction works for all even $m \geqslant 6$. This says that the strongly $\mathbf{Z}$-essential class is a wider class than the rational $K$-manifolds. In particular, in these constructed examples there are no connected Lie groups acting on $M^{m}$. For any $S^{1}$-action would have to be injective and so $\pi_{1}(M)$ would have to have nontrivial center which it does not have. 
(ii) It would be interesting to have a class of $\mathbf{Z}$-essential manifolds for which there does exist actions (which a fortiori will have to be injective toral actions) but for which this fact cannot be detected by (rational $K$-manifold $\Rightarrow$ injective toral action). This is easily obtained by just taking $M^{\prime}=M^{m} \times S^{1}$, where $M^{m}$ are the even dimensional manifolds just constructed. For $K^{\prime}$ we take $K(\pi, 1) \times S^{1},\left(\pi_{1}(M)=\pi\right)$. $M^{\prime}$ is Z-essential but not rationally a $K$-manifold. From the theory of injective actions [CR3], the effective actions on $M^{\prime}$ are circle actions which must be free product actions. However, it is possible, in the topological case, for the splitting of $M^{m} \times S^{1}$ by the action of $S^{1}$ to yield a nonmanifold for the orbit space (= global slice to a product action).

(iii) The construction in Example 4.4 also suggests a negative answer to a question of Browder and Hsiang [BH, 5.6].

In their example in $\S 5$ of $[\mathbf{B H}]$, a closed smooth $G$-manifold $D W$ with a smooth $S^{1}$-action is constructed for which in their terminology:

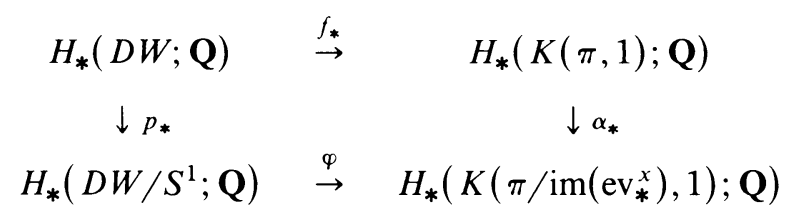

commutes, the homomorphisms are nontrivial in dimension 6 but there is no map $D W / S^{1} \rightarrow K\left(\pi / \operatorname{im}\left(\operatorname{ev}_{*}^{x}\right), 1\right)$ which induces $\varphi$. Since $D W$ admits both smooth noninjective as well as injective smooth $S^{1}$-actions, $D W$ cannot be an essential manifold.

They point out in their Question (5.6) that $H_{*}\left(K\left(\pi_{1}\left(D W / S^{1}\right), 1\right) ; \mathbf{Q}\right) \cong$ $H_{*}\left(K\left(\pi_{1}(D W) / \operatorname{im}\left(\mathrm{ev}_{*}^{x}\right), 1\right) ; \mathbf{Q}\right)$ and they ask whether it is true in general. (If it were true then the paper could be simplified.) However, this, for essential manifolds, is often not true. For an easy low dimensional example consider any Seifert manifold $\Sigma^{3}$ which is a rational homology sphere with infinite fundamental group. $\Sigma^{3}$ is a $K\left(\pi_{1}\left(\Sigma^{3}\right), 1\right)$ and it admits a unique, up to equivariant diffeomorphism, $S^{1}$-action with orbit space the 2-sphere. $\pi_{1}\left(\Sigma^{3}\right) / \operatorname{im}\left(\operatorname{ev}_{*}^{x}\right)=\Gamma$, a Fuchsian or Euclidean crystallographic group which is normally generated by finite cyclic groups. Now, $H_{2}(\Gamma ; \mathbf{Q})$ $\approx \mathbf{Q}$, but $\pi_{1}\left(\Sigma^{3} / S^{1}\right)=\pi_{1}\left(S^{2}\right)=1$.

4.6. Admissible $\Rightarrow$ Essential.

EXAMPLE. Let $M_{i}$ be 3-dimensional spherical space forms, $\pi_{1}\left(M_{i}\right)=A_{i}$ (finite) not cyclic $(i=1,2)$ and $A_{1} \neq A_{2}$. Let $M=M_{1} \# M_{2}$. Then $M$ is admissible but is not essential.

We shall give two separate arguments. The first one requires smoothness. First, observe that $M$ is not essential because $H^{3}\left(A_{1} * A_{2} ; \mathbf{Q}\right)=\oplus H^{3}\left(A_{i} ; \mathbf{Q}\right)=0$. In fact, $H^{3}\left(A_{1} * A_{2} ; \mathbf{Z}\right)$ has rank 0 . Now, suppose $M$ was not smoothly admissible. Then there exists some $\mathbf{Z}_{p}$ ( $p$ prime) acting on $\tilde{M}$ smoothly and commuting with $\pi=A_{1} * A_{2}$. Since $\pi_{2}(M) \neq 0$, there exists a smoothly embedded $\mathbf{Z}_{p}$-equivariant 2-sphere $S$ representing a nonzero element of $\pi_{2}(M)$ by Meeks-Yau [MY]. In fact, $M=M_{1} \# M_{2}$ where the connected sum is taken along the $\mathbf{Z}_{p}$-equivariant $S$. The $\mathbf{Z}_{p}$ 
action extends smoothly to $M_{i}$ by coning over the 2 -sphere $S$. (The $\mathbf{Z}_{p}$ action cannot interchange the $M_{i}$ 's since $A_{1} \neq A_{2}$.) Since each $\left(\mathbf{Z}_{p}, M_{i}\right)$ has fixed points, the action can be lifted to the $S^{3}=\tilde{M}_{i}$, the universal covering. The lifted action commutes with $A_{i}$, since $\mathbf{Z}_{p}$ acts trivially on $A_{1} * A_{2}$.

Since $M_{i}$ does not admit any orientation-reversing self-homotopy equivalence (see [NR, §8]), $\mathbf{Z}_{p}$ must be orientation preserving and so $\operatorname{Fix}\left(\mathbf{Z}_{p} ; S_{i}^{3}\right)=S^{1}$. Because $\mathbf{Z}_{p}$ commutes with $A_{i}, A_{i}$ acts on the fixed point set as a covering group. But this is impossible because $A_{i}$ is not cyclic, cf. [CR2, A10].

The second argument uses less machinery, does not require smoothness, and extends somewhat to higher dimensions. Let $M=M_{1} \# M_{2}$ where $M_{i}$ are spherical space forms not $S^{3}$ or $\mathbf{R} P_{3}$. The universal covering $\tilde{M}$ is homeomorphic to $S^{3}$ with a totally disconnected set $C$, the ends of $\tilde{M}$, deleted from $S^{3}$. The $\mathbf{Z}_{p}$ action extends to $S^{3}$ as does the covering $A_{1} * A_{2}$ action. Because the $\mathbf{Z}_{p}$ action commutes with $\pi_{1}(M)$, the extended $\mathbf{Z}_{p}$ action fixes the ends of $C$, see [B1, 2.3.1]. Suppose $\mathbf{Z}_{p}$ preserves the orientation, so $\operatorname{Fix}\left(\mathbf{Z}_{p}, S^{3}\right)=S$, a 1-sphere. This 1-sphere is $A_{1} * A_{2}$ invariant. For, $g(\sigma(t))=\sigma(g t)=\sigma(t), t \in S, g \in \mathbf{Z}_{p}, \sigma \in A_{1} * A_{2}$.

Now consider the action of $A_{1}$ on $S$. Since it is free on $S-C, A_{1}$ must be cyclic or dihedral. But $\pi_{1}\left(M_{i}\right)=A_{i}$ cannot be dihedral. So $A_{1}$ and $A_{2}$ must both be cyclic. Consequently, if neither $M_{i}$ are lens space then $M$ is admissible. Similarly, if $\mathbf{Z}_{p}$ reversed orientation, then $p=2$, and $\operatorname{Fix}\left(\mathbf{Z}_{2}, S^{3}\right)=S^{2}$. Since $A_{i}$ acts effectively on $S^{2}$, and $A_{i}$ is $\pi_{1}\left(M_{i}\right), A_{i}$ must be cyclic. Q.E.D.

We should observe that on a connected sum of lens spaces there are actions of $\mathbf{Z}_{p}$ that commute with $\pi_{1}(M)$ and which lift to the universal covering. Just take an $S^{1}$-action on $M$. It will have fixed points. Lift this action to $\tilde{M}$ and take $\mathbf{Z}_{p} \subset S^{1}$, with $p$ not dividing the orders of $\pi_{1}\left(L_{i}\right)$. So $M$ would not be admissible nor almost weakly admissible (cf. 3.5).

4.7. Every smooth inner action is abelian $\nRightarrow$ Almost weakly admissible/Injective. Let $M=M_{1} \# M_{2}$ where $M_{i}$ are 3-dimensional lens spaces such that $M_{i}$ do not admit orientation reversing homeomorphisms and $\pi_{1}\left(M_{1}\right)=A_{1} \neq A_{2}=\pi_{1}\left(M_{2}\right)$.

EXAMPLE. $M$ satisfies the property that every smooth inner action is abelian but $M$ is not almost weakly admissible. Moreover, every connected compact group of homeomorphism is a circle and has fixed points.

We have just seen that $M$ is not almost weakly admissible. Our task is to show that every smooth inner action on $M$ is abelian. The theorem of Meeks and Yau [MY] implies that if $G$ acts smoothly and effectively on $M$ then $G$ preserves orientation, and is isomorphic to a subgroup of $S O(3)$.

The lifting sequence $1 \rightarrow \pi_{1}(M) \rightarrow E \rightarrow G \rightarrow 1$ yields an action of $E$ on $\tilde{M}$. Because $G$ is inner and $Z\left(\pi_{1}(M)\right)=1$, the group $E$ is isomorphic to $\pi_{1}(M) \times G \subset$ $\mathscr{H}(\tilde{M})$. Just as in 4.6 , the action of $G$ extends trivially to the ends $C$ of $\tilde{M}$. Moreover, the endpoint compactification $\tilde{M} \cup C$ is homeomorphic to the 3-sphere $S^{3}$.

We shall show that $G$ contains no dihedral subgroup and so $G$ must be cyclic, and the action would be inner. Suppose $G$ contains a dihedral group $\mathbf{Z}_{n} \rtimes \mathbf{Z}_{2}$. $\operatorname{Fix}\left(\mathbf{Z}_{n}, S^{3}\right)$ is a 1-sphere, $S$, because we are dealing with a 3-dimensional manifold. Note $C \subset S$, 
and that $S$ is also $\mathbf{Z}_{2}$-invariant, $\left(h g(x)=g\left(g^{-1} h g(x)\right)=g\left(h^{-1}(x)\right)=g(x)\right.$ for $\left.g \in \mathbf{Z}_{2}, g \neq e, h \in \mathbf{Z}_{n}, x \in S\right)$. Consequently, $S=\operatorname{Fix}\left(\mathbf{Z}_{2}, S^{3}\right)$ and hence $S=$ $\operatorname{Fix}\left(\mathbf{Z}_{n} \rtimes \mathbf{Z}_{2}, S^{3}\right)$. Choose $y_{0} \in S-C$. The projection of $y_{0}$ to $x_{0} \in M$ is also fixed by $\mathbf{Z}_{n} \rtimes \mathbf{Z}_{2}$. Therefore, $\mathbf{Z}_{n} \rtimes \mathbf{Z}_{2} \subset G$ acts smoothly on $M$ fixing $x_{0}$. We can choose a small smooth $\mathbf{Z}_{n} \rtimes \mathbf{Z}_{2}$-invariant ball on which $\mathbf{Z}_{n} \rtimes \mathbf{Z}_{2}$ acts linearly and which lifts to a smooth $\mathbf{Z}_{n} \rtimes \mathbf{Z}_{2}$-invariant ball neighborhood $B$ of $y_{0}$ in $\tilde{M}$. But $y_{0} \in S \cap B$ is not isolated and so $G$ could not contain $\mathbf{Z}_{n} \rtimes \mathbf{Z}_{2}$ for any $n$.

For the remaining possibilities, $G$ could be isomorphic to the tetrahedral, octahedral or icosahedral group. But each of these groups contains $\mathbf{Z}_{2} \times \mathbf{Z}_{2}$ as a subgroup and so $G$ must be cyclic. Q.E.D.

The only connected compact groups that can act effectively on the nontrivial connected sums of lens spaces is the circle. Each action has fixed points, see [R]. This shows that smooth inner actions are abelian $\nRightarrow$ injective.

4.8. Torus $\Rightarrow$ Inner actions are abelian/Injective.

EXAMPLE. $M=\left(S^{2} \times S^{1}\right) \#\left(S^{2} \times S^{1}\right)$ admits no compact connected group action other than the circle with fixed points. Since the circle action has fixed points, it cannot be injective. Further, $M$ admits a dihedral inner action.

$M$ admits two $S^{1}$-actions up to topological conjugacy [R]. A compact connected group acting on a 3-manifold must be a Lie group and one can easily show that only the circle acts on $M$ among these. The two actions can easily be described as follows. Take either the 3 times punctured sphere or the once punctured torus. Form the product with $S^{1}$ and collapse each $S^{1}$ orbit over each boundary point to a single point. In both cases, the surface with boundary can be identified with a global cross section to the action and the boundary identified with the fixed point set. Now using the global cross section, take the usual action of the dihedral group $\mathbf{Z}_{p} \rtimes \mathbf{Z}_{2}$ on each circle fiber and extend to be trivial over the fixed point set. For the 3 times punctured sphere the bounding curves generate $\pi_{1}(M)$. These curves are fixed under all elements of $\mathbf{Z}_{p} \rtimes \mathbf{Z}_{2}$. So this action must be inner since it induces trivial automorphisms on $\pi_{1}(M)$.

4.9. Smoothly injective $\nRightarrow$ Smooth inner actions are abelian (and hence, not weakly admissible).

EXAMPLE. Let $M$ be a 4-dimensional complex manifold satisfying $Z_{0}^{4}+Z_{1}^{4}+Z_{2}^{4}$ $+Z_{3}^{4}=0$ in $\mathbf{C} P^{3}$. This is known as a $K_{3}$-surface. It is a simply connected spin 4-manifold with its first Pontrjagin class nonzero. Therefore, every smooth $S^{1}$-action is trivial since its $\hat{A}$-genus is nonzero [AH]. A fortiori, then, every effective smooth toral action on $M$ is injective. However, the symmetric group $S_{4}$ acts smoothly and effectively on $M$ by permuting the variables in $\mathrm{CP}^{3}$. This action is inner since $M$ is simply connected. Similarly, $M$ could not be weakly admissible. It seems plausible that $M$ is smoothly almost weakly admissible but that the smoothness assumption cannot be dropped on $M$ itself.

ADDED IN PROOF. Recent results announced by S. Kwasik and R. Schultz imply that if $M$ is a closed connected topological spin 4-manifold (such as the $K_{3}$-surface) and admits a topological circle action, then the signature of $M$ is 0 . They have also shown that every closed simply connected topological spin 4-manifold admits topological cyclic group actions of arbitrary finite order. 
5. Summary. We summarize in a diagram the refinements of Theorem 1 and the examples of Theorem 2 .

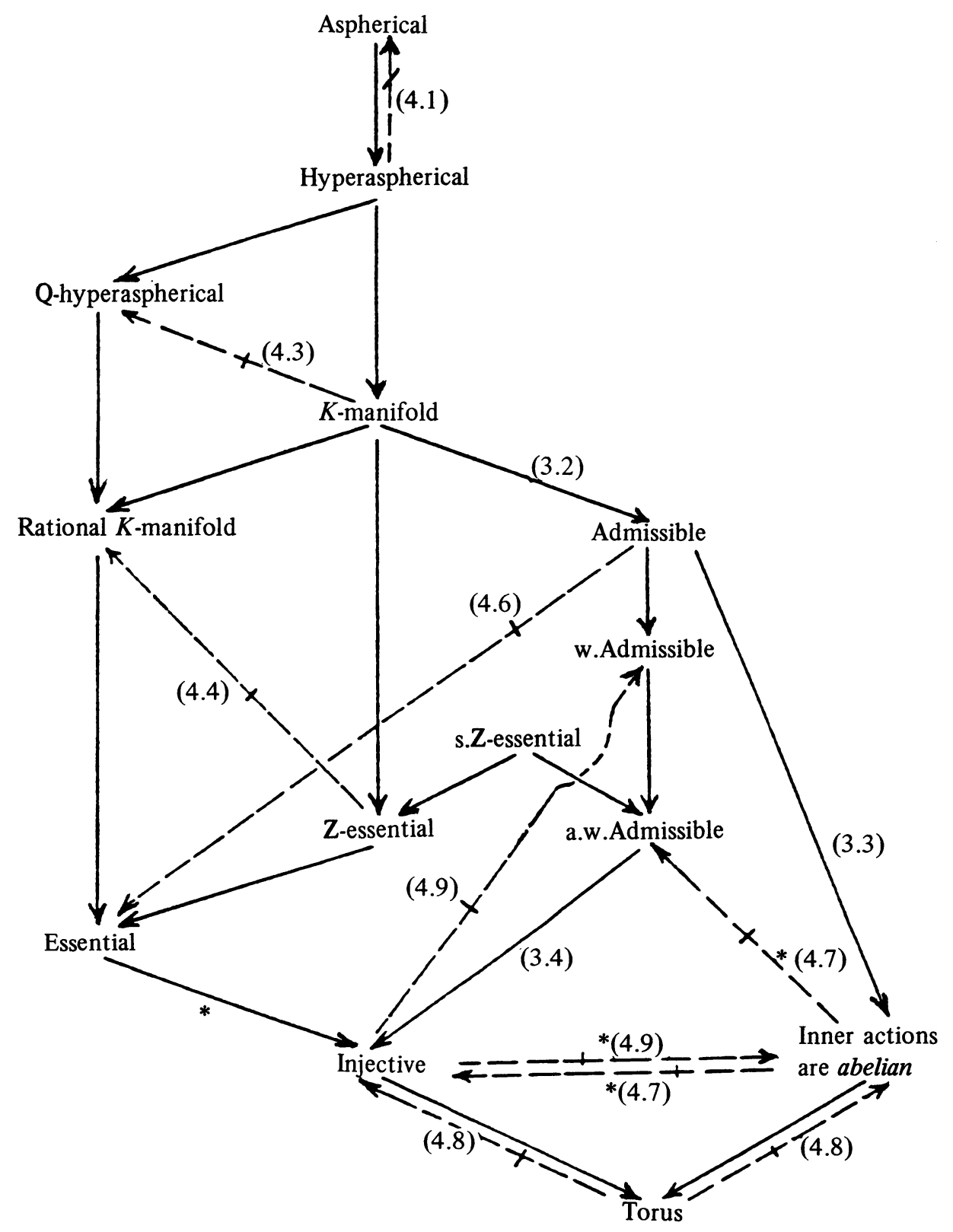

* indicates a smoothness assumption. $M_{1} \# M_{2}, \quad M_{1}$ aspherical, $M_{2}$ not aspherical,

$$
f: M \rightarrow K(\pi, 1) \cong_{\text {(homology) }} \mathbf{C} P_{m},
$$$$
f: M \rightarrow K(\Gamma, 1), \quad \pi_{1}(M)=Q \times Q \times Q
$$ 
(4.6) $\quad M=M_{1} \# M_{2}, \quad M_{i}$ spherical space form which is not a lens space,

$$
\begin{gathered}
M=M_{1} \# M_{2}, \quad M_{i} \text { is a lens space, } \\
M=S^{2} \times S^{1} \# S^{2} \times S^{1} \\
K_{3} \text {-surface. }
\end{gathered}
$$

\section{BIBLIOGRAPHY}

[A] M. A. Armstrong, Calculating the fundamental group of an orbit space, Proc. Amer. Math. Soc. 84 (1982), 267-271.

[AB] A. Assadi and D. Burghelea, Examples of asymmetric differentiable manifolds, Math. Ann. 255 (1981), 423-430.

[AH] M. Atiyah and F. Hirzebruch, Spin-manifolds and group actions. Essay on topology and related topics, Springer, Berlin and New York, 1970, pp. 18-28.

[B1] E. M. Bloomberg, Manifolds with no periodic homeomorphisms, Trans. Amer. Math. Soc. 202 (1975), 67-78

[BDH] G. Baumslag, E. Dyer and A. Heller, The topology of discrete groups, J. Pure Appl. Algebra 16 (1980), 1-47.

[BH] W. Browder and W. C. Hsiang, G-actions and the fundamental group, Invent. Math. 65 (1982). 411-424.

[C] P. E. Conner, Differentiable periodic maps, 2nd ed., Lecture Notes in Math., vol. 738, Springer, 1979.

[CR1] P. E. Conner and Frank Raymond, Actions of compact Lie groups on aspherical manifolds, Topology of Manifolds (Proc. Inst., Univ. of Georgia, Athens, 1969), Markham, Chicago, Ill., 1970, pp. $227-264$.

[CR2] , Manifolds with few periodic homeomorphisms, Proc. Second Conference on Compact

Transformation Groups, Part II, Lecture Notes in Math., vol. 299, Springer, 1972, pp. 1-75.

[CR3] Injective actions of toral groups, Topology 10 (1970), 283-296.

[CR4] Deforming homotopy equivalences to homeomorphisms in aspherical manifolds, Bull. Amer. Math. Soc. 83 (1977), 36-85.

[CR5] _ Holomorphic Seifert fiberings, Proc. Second Conference on Compact Transformation Groups, Part II, Lecture Notes in Math., vol. 299, Springer, 1972, pp. 124-204.

[DS] H. Donnelly and R. Schultz, Compact group actions and maps into aspherical manifolds, Topology 21 (1982), 443-455.

[F] E. Floyd, Orbits spaces of finite transformation groups. II, Duke Math. J. 22 (1955), 33-38.

[GLO] D. Gottlieb, K. B. Lee and M. Ozaydin, Compact group actions and maps into $K(\pi, 1)$-spaces, Trans. Amer. Math. Soc. 287 (1985), 419-429.

[Gr] M. Gromov, Volume and bounded cohomology, Inst. Hautes Etude Sci. Publ. Math. 56 (1982), 213-307.

[KK] H. T. Ku and M. C. Ku, Group actions on aspherical $A_{k}(N)$-manifolds, Trans. Amer. Math. Soc. 278 (1983), 841-859.

[LR1] K. B. Lee and F. Raymond, Topological, affine and isometric actions on flat Riemannian manifolds, J. Differential Geom. 16 (1982), 255-269.

[LR2] Geometric realization of group extensions by the Seifert construction, Contemporary Math., vol. 33, Amer. Math. Soc., Providence, R. I., 1984, pp. 353-411.

[LY] H. B. Lawson and S. T. Yau, Compact manifolds of non-positive curvature, J. Differential Geom. 7 (1972), 211-228.

[MY] W. Meeks and S. T. Yau, Topology of three-dimensional manifolds and the embedding problems in minimal surface theory, Ann. of Math. 112 (1980), 441-484.

[NR] W. Neumann and F. Raymond, Seifert manifolds, plumbing, $\mu$-invariant and orientation reversing maps, Alg. and Geom. Topology (Proc. Santa Barbara, 1977), Lecture Notes in Math., vol. 664, Springer, 1978, pp. 163-196. 
[Sch1] R. Schultz, Group actions on hypertoral manifolds. I, Topology Symposium (Siegen 1979), Lecture Notes in Math., vol. 788, Springer, pp. 364-377.

[Sch2] __, Group actions on hypertoral manifolds. II, J. Reine Angew. Math. 325 (1981), 75-86.

[Sp] E. Spanier, Algebraic topology, McGraw-Hill, 1966.

[SY] R. Schoen and S. T. Yau, Compact group actions and the topology of manifolds with non-positive curvature, Topology 18 (1979), 361-380.

[WW] R. Washiyama and T. Watabe, On the degree of symmetry of a certain manifold, J. Math. Soc. Japan 35 (1983), 53-58.

Department of Mathematics, University of OKlahoma, Norman, OKLahoma 73019

Department of Mathematics, University of Michigan, Ann Arbor, Michigan 48109 